\title{
Performance in a delayed comparison discrimination task as a function of stimulus interpretation
}

\author{
GREGORY W. CERMAK \\ Stanford University, Stanford, California 94305
}

\begin{abstract}
In a sequential same-different comparison task, physically similar visual stimuli with the same interpretation were more poorly discriminated than equally similar stimuli with different interpretations. Physical similarity of stimulus pairs was held constant by using parametrically controlled computer-generated visual forms and by using each pair of figures as both a sameinterpretation pair and as a different-interpretation pair. The interpretation variable used the ambiguous-figure properties of the forms. The two forms in each pair could be seen as representing the same object given one way of interpreting them; a second interpretation applied to only one member of the pair. The discrimination results held for interstimulus intervals of $300 \mathrm{msec}$ and 13 sec. Similarities between the present studies and "categorical perception" studies are noted.
\end{abstract}

Although much of human cognitive activity involves the interpretation of stimuli, relatively few studies have explicitly used interpretation as an independent variable (cf. Hochberg, 1968). Instead, the interpretation of stimuli has been treated as a dependent variable, as, for example, in the study of visual illusions and ambiguous figures (cf. Bugelski \& Alampay, 1961), or has been treated as a process rather than as a variable, as in the pattern recognition literature (e.g., Uhr, 1966). When the interpretation of a stimulus has been viewed as an independent variable, it has been found (as might be expected) to be an important part of what is encoded or retrieved concerning a stimulus. For example, Bower, Karlin, and Dueck (1975) showed that nonsense pictures are recalled better if they have been given labels which facilitate their interpretation. Sounds with more than one plausible interpretation are recognized better if they have the same labels during study and test (Bower \& Holyoak, 1973). Hochberg (1968) points out that many perceptual phenomena, such as geometrical illusions and apparent motion, depend on prior interpretation of the stimuli. Studies of "categorical perception" have

Experiments 1 and 2 were completed while the author held an NDEA predoctoral fellowship, and were part of a doctoral dissertation submitted to the faculty of Stanford University. Computer use was made possible by National Science Foundation Grant GB-31971X to Roger Shepard, the author's dissertation advisor. Thanks are expressed to Ernest Hilgard and James Juola for use of equipment, and to James Juola, Sally Springer, and Brad Southworth for reading an earlier version of the manuscript. Special thanks go to Roger Shepard for valuable advice and suggestions. The author's present address is: Societal Analysis Department, Research Laboratories, General Motors Corporation, 12 Mile and Mound Roads, Warren, Michigan 48090. indicated that, for some types of stimuli, the interpretation of the stimulus is nearly all that is encoded (cf. Liberman, Mattingly, \& Turvey, 1972, p. 320).

The "categorical perception" studies have, for the most part, been viewed as demonstrating an unusual phenomenon, and differences between these studies and other areas of information processing have been emphasized (cf. Studdert-Kennedy, Liberman, Harris, \& Cooper, 1970). The present paper examines some features that categorical perception studies have in common with other studies which use the interpretation of stimuli as an independent variable. A general classification of types of experiments is offered, and three experiments are reported which bear on the generality of some aspects of categorical perception results.

Given the distinction between interpretation as an independent variable and interpretation as a dependent variable, there are still many possible theoretical frameworks in which interpretation of stimuli may be placed. As examples, there are models of the perceiver, such as the information-processing models and physiological models, and there are approaches which do not deal directly with the perceiver, but instead stress the properties of the stimuli perceived (e.g., Garner, 1972) or use an inputoutput approach which assumes little about the perceiver. The present approach to interpretation will concentrate primarily on simple characteristics of the stimuli, or observable reactions to stimuli, rather than on models of the perceiver's processes or structures.

In particular, the basic observation here is that the interpretation of members of a set of stimuli partitions them into equivalence classes, each class or category being composed of stimuli with the "same", interpretation. In the present case, then, the per- 
ceiver is viewed merely as an operator on a set of stimuli. The degree of "sameness" of the interpretations of two stimuli might be viewed as a function of (a) the number of stimuli which are grouped in a particular class by virtue of their interpretation and (b) whether classes have internal structure. While class size and structure and interclass relationships (such as nested and overlapping classes) could be cast as independent variables, the questions addressed here concern only class membership. What is the effect of a stimulus being placed in one class rather than in another? Or, what is the effect of a stimulus having one interpretation rather than another? The question is not whether a stimulus belongs to some class, but to which class. Thus, the independent variable of concern inherently involves at least two classes or interpretations.

Several elementary approaches are possible for producing an independent variable which captures the idea of a stimulus being in one class rather than in another. In the most elementary approach, the same physical stimulus would belong at different times to two different classes, as in the case of a stimulus which can reasonably be interpreted in two different ways. The next most elementary independent variable would use two stimuli, at least one of which would have to belong to at least two classes. The relationship of the two stimuli (same class or different classes) would serve as the independent variable. A slightly more complicated, though still basic, approach, given a minimum of two classes, would be to let the relationship of three stimuli $(a, b, x)$ be the independent variable: Two stimuli $(a, x)$ could be in one class and one $(b)$ in the other class; except for class membership, all other relations between the members of the pairs $(a, x)$ and $(b, x)$ would have to be the same for the two pairs. If no stimulus can have more than one interpretation, the three-stimuli arrangement is the simplest one which allows class membership to be a variable while holding other aspects of the stimuli constant.

Studies using a variation of the basic singlestimulus-with-multiple-interpretations paradigm have focused primarily on encoding and memory effects (e.g., Bower \& Holyoak, 1973; Bower et al., 1975; Hochberg, 1968; Riley, 1962). The general conclusion from such studies is that interpretation is a process of organizing, structuring, and often reducing sensory information according to strategies which are often under cognitive control. Information encoded in a structured way is easier to retrieve (Bower \& Holyoak, 1973; Bower et al., 1975). In the class terminology, the interpretation process produces classes of stimuli upon encoding, and information concerning class membership is effective in retrieval. However, class information is not all that is encoded, and there does not appear to be evidence that class information interferes with other information about the stimuli, such as their finegrained physical characteristics (cf. Hochberg, 1968; Riley, 1962).

Studies using the two- and three-stimuli methods have produced conclusions quite different from those from the single-stimulus experiments, despite their formal similarity. Multiple-stimuli experiments, constrained to use the relationship of stimuli as the independent variable, have usually chosen confusability of the stimuli as the dependent variable. In the main family of multiple-stimulus interpretation studies, the "categorical perception" studies, conclusions have been drawn which differ from conclusions drawn in the single-stimulus experiments either in their account of the subject's informationprocessing abilities or in their attribution of special properties to the stimulus set employed (e.g., Mattingly, Liberman, Syrdal, \& Halwes, 1971). Because the particular type of stimuli employed in the categorical perception studies plays such a central role, it is of interest to determine whether similar results are obtainable with stimuli from other domains.

The independent variable of the original categorical perception studies was defined basically on groups of three stimuli, two of which were in the same class, and one of which was in a different class (Liberman, Harris, Hoffman, \& Griffith, 1957). The stimuli were synthetic speech sounds, often the stop consonants $/ b /, / d /, / g /$, which happen to be describable as a constant acoustic component plus a variable component, which can be represented in one physical dimension (Liberman, Cooper, Shankweiler, \& Studdert-Kennedy, 1967). Thus, the stimuli varied in one natural dimension and differences between the stimuli were measurable in terms of the physical dimension. The differences between the middle stimulus and the two end stimuli of each triple were in most respects identical except with respect to class membership. The dependent variable was discrimination accuracy for each of several pairs of stimuli (where each pair was embedded in a triple). Stimuli in the same class were discriminated much more poorly than stimuli in different classes. A stronger result was that discrimination accuracy could be fairly well predicted by identification probability (e.g., Liberman et al. 1957; also see Cutting \& Rosner, 1976).

One possible indication from such discrimination results is that stimulus class membership has the inherent property that members of the same class are more easily confused than members of different classes, other things being equal. Naturally, the single stimulus experiments would be incapable of showing such a result, but it is possible that the general information-processing principles which are affirmed by the single-stimulus interpretation studies would also hold for the multiple-stimulus 
interpretation studies. However, it was argued in the early categorical perception studies that these discrimination results should apply only to speech stimuli, because the phenomenon was produced by an interaction between special speech production and speech perception processing routines (cf. Lane, 1965; Studdert-Kennedy et al., 1970). Now a distinction is made between strong results, in which discrimination is predicted well by interpretation probabilities, and weaker results, in which predictions are not as accurate. The strong results apply to consonants, and weaker results are found with speech sounds generally (e.g., Pisoni, 1975). Present concern is with the weaker and more general results.

Several questions concerning the original categorical perception results have provoked further research while bears on the present studies: (1) Is the phenomenon indeed limited to speech sounds? (2) Does the original experimental procedure guarantee the results, independent of stimulus set? (3) Is the phenomenon more properly a memory effect, rather than a perception effect? (4) If the effect is not limited to speech sounds, is it limited to auditory stimuli?

Cutting and Rosner $(1974,1976)$ demonstrated poorer discrimination of stimuli in the same class, as compared to stimuli in different classes, using musical sounds as stimuli. Cross, Lane, and Sheppard (1965) also demonstrated a connection between discrimination and class membership, but using visual stimuli. Both studies used the ABX discrimination test which was originally used in categorical perception demonstrations, and which became part of the operational definition of categorical perception (cf. Liberman et al., 1957; Studdert-Kennedy et al., 1970). The ABX procedure is to present stimulus $\mathbf{A}$, wait one or more seconds, present stimulus B (different from A), wait, then present an unknown stimulus, either A or B, which the subject must identify. Cross et al. (1965, p. 73) argued (in different terminology) that the ABX procedure includes a time component which affects information about class membership (i.e., binary response cues) less than it affects information about the physical identity of the stimulus. Stimuli from the same class, differing only in physical identity, would naturally be confused more if the physical information were degraded over time. Cutting and Rosner (1974), among several others now, have proposed essentially the same memory account of performance in an $A B X$ task. Thus, it is not clear that the ABX task results show a discrimination decrement for nonspeech stimuli in the same class.

Experiments by Pisoni show that the discrimination effect for speech sounds occurs using other discrimination tests, some of which also suggest that a discrimination effect may be separable from the memory effect. Pisoni (1973) used machine-produced consonants in a delayed comparison recognition memory test to determine stimulus discriminability. Two stimuli were presented on each trial, with a delay interval between the stimuli of 0 to $2.0 \mathrm{sec}$. The response required was "same" or "different." The discrimination effect was obtained: When stimuli were in the same class (had the same interpretation), they were discriminated more poorly than when they were in different classes. This effect was greater at longer delays, but the basic effect was apparent at the 0-sec delay. Pisoni and Tash (1974) replicated the discrimination results at .25 -sec delay in another same-different discrimination task using reaction time as the response measure. Apparently, the within-between class discrimination effect is to some degree an encoding effect or response effect, rather than solely an effect associated with storage time between encoding and response.

It is still not clear whether or not the weak discrimination effect is limited to speech sounds, because of the use of the ABX procedure in the demonstrations involving other stimuli. However, it is possible that general and elementary characteristics of auditory encoding, masking, and memory might account for the effect (cf. Pisoni, 1973). Whether characteristics of visual processing, or of human information processing in general, could account for within-between class discriminability differences is an open theoretical question. Empirically, it has not been demonstrated that stimulus class membership is correlated with the discriminability of visual stimuli, independent of the ABX procedure or of other memory effects (cf. Cross et al., 1965; Lenneberg, 1961).

The experiments reported here had the following features: (a) Stimulus interpretation served as an independent variable which defines stimulus class membership. (b) The basic stimulus unit was the pair, both of whose members could have multiple interpretations. (c) The independent variable defined on these pairs was a relationship, i.e., the stimuli had the same interpretation or they did not. (d) The stimuli were visual. (e) Stimulus confusability ("discriminability"') served as the dependent variable. (f) Confusability was measured (without the ABX technique) under two delay conditions in a sequential presentation same-different recognition task.

\section{Suitable Stimuli}

As mentioned before, very special properties are required of the stimulus pairs when using interpretation as an independent variable: The stimuli should be amenable to arrangement in pairs so that the physical similarity of stimuli with different interpretations is the same as the physical similarity of stimuli with the same interpretation. A set of visual stimuli with the desired properties is available, and is described in detail in Shepard and Cermak (1973). 
The figures individually tend to look like caricatures of common objects, so that most figures have natural interpretations. Each figure is described by the same mathematical expression, which has two parameters, each of which happens to assume one of nine possible values. The total possible stimulus set can be viewed as two-dimensional, and the particular stimulus set sampled consists of 81 equally spaced points in the parameter space. Physical similarity of the figures changes progressively with change in the parameters, so that differences in the generating parameters can be taken as measuring (some) physical differences of the stimuli. By interpreting the stimuli, however, the stimulus space becomes partitioned into several regions, within each of which all the stimuli can assume the same interpretation. There are rather sharp "boundaries" between the regions, in the sense that the difficulty in interpreting stimuli in a particular way increases more rapidly per unit of difference in the underlying stimulus-generating parameters around a boundary. Many of the figures have the further property that they may be interpreted in more than one way, i.e., they are "ambiguous" (see Figure 1). The partition induced on the stimulus set by one interpretation generally is not the same as the partition induced by some other interpretation. In particular, the region in the stimulus parameter space associated with one interpretation can partially overlap the region associated with another interpretation (see Figure 2). An independent variable, class membership or sameness of interpretation, can be defined using the basic two-stimulus paradigm: Under one interpretation, the two stimuli are in the same class; under a second interpretation they are in different classes. Stated differently, two stimuli may have the same interpretation, but a second interpretation may apply to only one of the pair.

A slightly more complicated variable of the same basic type can be defined on three stimuli, T,C1, C2. Under one interpretation, $T$ and $C 1$ might be in the same class, and $\mathrm{T}$ and $\mathrm{C} 2$ in different classes, but under another interpretation, $\mathrm{T}$ and $\mathrm{C} 2 \mathrm{might}$ be in the same class and $\mathrm{T}$ and $\mathrm{Cl}$ now in different classes (see Figure 2). By choosing $\mathrm{Cl}$ and $\mathrm{C2}$ to be equidistant from $\mathrm{T}$ in the parameter space, physical similarity of $(T, C 1)$ and $(T, C 2)$ can be held constant (to some approximation), as in the basic three-stimulus paradigm. It should be noted that the real unit of the independent variable is the pair ( $T, C x)$ under two interpretations; physical similarity is held constant while class inclusion is manipulated.

By what criterion should the interpretation of two stimuli be considered the same or different? Clearly, if the interpretations were indistinguishable (to someone), they would be considered the same. But "indistinguishable" requires definition in terms of confusability or discriminability-which is to be

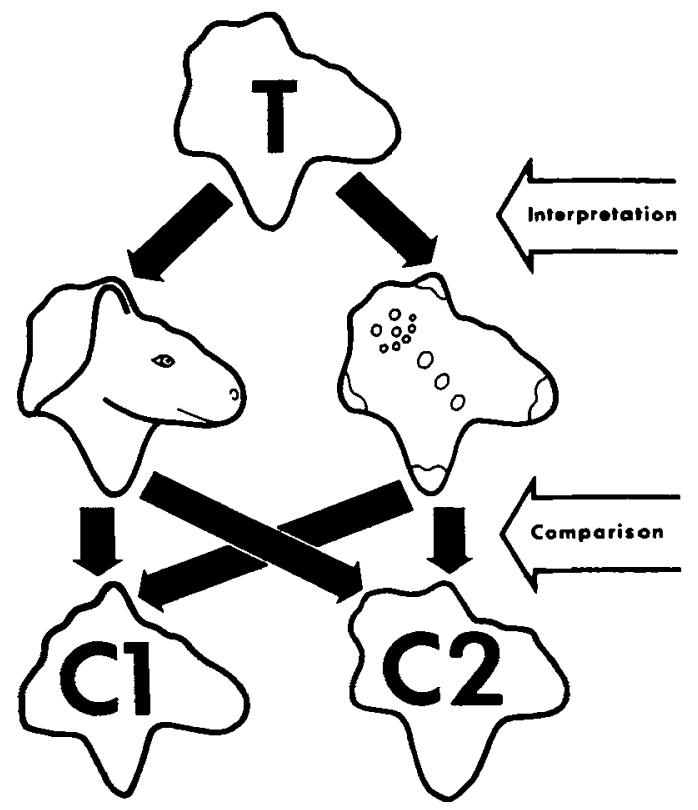

Figure 1. One of the eight target-comparison triples (T,C1,C2). $T$ is compared to $C 1$ and $C 2$ under two different interpretations. In the terminology of the Shepard and Cermak (1973) paper, $\mathrm{T}$ is $\mathrm{B2}, \mathrm{C1}$ is $\mathrm{C1}$, and $\mathrm{C2}$ is $\mathrm{A3}$.

the dependent variable. Another possible criterion involves the number of stimuli which are allowed in the same class. It might be argued that if the class is very large, then "sameness" of interpretation, so defined, is meaningless. Whether or not a classification is meaningless can be evaluated empirically by its power as an independent variable. Thus, the advisability of using a particular criterion (for calling

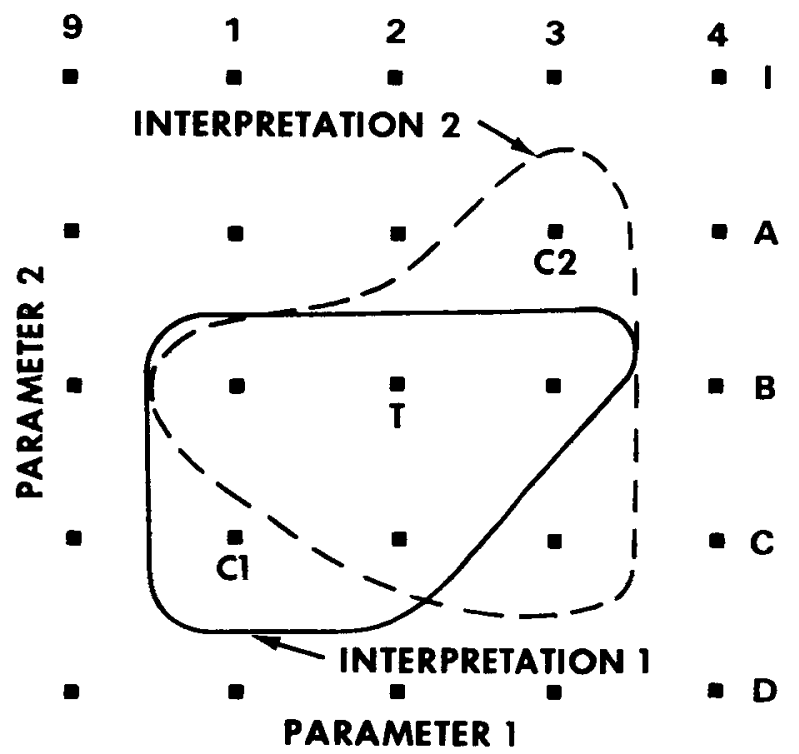

Figure 2. A target-comparison triple placed in the figuregenerating parameter space, with empirical isosimilarity contours for two interpretations. Numbers and letters identifying points in the parameter space correspond to those used in Shepard and Cermak (1973). 
two interpretations the same) is to be determined a posteriori.

Various measures or indicators of the interpretation of a stimulus might be considered. The indicator chosen was that a stimulus be classified with respect to a standard. If it was "easy to see the same sort of object in the [ambiguous] figure as that portrayed by the [easily interpretable] target," the figure was counted as belonging to the same class as the target, otherwise not. In Experiment 1, collections of stimuli which could be interpreted the same (in the sense of "same" described above) were defined. With this information, a prediction was made: that stimuli with the "same" interpretation would be distinguished more poorly than stimuli with different interpretations in a yes-no sequentialpresentation recognition test. Experiments 2 and 3 tested this prediction for two different time delays between the stimuli. The "ambiguous figure" nature of the stimuli was used to guarantee that the parametric difference between "same interpretation" stimuli was identical to the parametric difference between "different interpretation" stimuli.

\section{EXPERIMENT 1: INTERPRETATION OF THE STIMULI}

In order to exploit the ambiguous-figure property of the drawings, enough actual instances must be found in which different interpretations lead to suitably different classes in the parameter space. In particular, a set of triples of figures $(T, C 1, C 2)$ was sought such that $T$ and $C 1$ (but not $C 2$ ) would fall within the same class under one interpretation and $T$ and $C 2$ (but not $\mathrm{C} 1$ ) would fall within the same class under another interpretation (as in Figure 2).

\section{Method}

\section{Stimuli}

The stimuli were the 81 outline figures described in Shepard and Cermak (1973). The particular interpretations used also were derived from that study. Nine figures, selected to cover the parameter space fairly uniformly, were each supplied with two different interpretations. This was done by drawing in extra lines to suggest some object. For example, a particular outline figure could be made to resemble a gingerbread man with one set of lines or an animal's head with another set (as in Figure 1). The outline alone could be "seen" either way. The outlines which had lines added (targets) and the plain outlines (comparison figures) were reproduced as individual $5 \times 5 \mathrm{~cm}$ black line drawings on $7.5 \times 7.5 \mathrm{~cm}$ pieces of white paper. The target and comparison figures, and the interpretations for each target, are listed in Table 1 according to their designations in Shepard and Cermak (1973).

\section{Subjects}

Forty-four students at Stanford University participated in the experiment as part of an introductory psychology course requirement. Nineteen subjects saw one of the two interpretations for each target; 25 saw the other interpretations of the targets. The subjects were tested in groups of about 5.

\section{Procedure}

Each subject was given a set of instructions, a set of the 81 figures, one of the two sets of nine target figures, and a response coding sheet. 'Two sets of nine targets each were prepared, so that each of the nine target outlines was included in each set, but the drawings on the targets in one set differed from the drawings on the targets in the other set. The instructions specified that one target figure at a time was to be used as a standard, and that each of the $\mathbf{8 1}$ plain figures, one at a time, was to be compared to the target. In each comparison, a judgment was to be made to assign the plain figure to one of three categories: (1) those figures for which "it is easy to see the same sort of object in the figure as that portrayed by the target," (2) figures for which the subject experienced "slightly greater difficulty in seeing the same object as in the target," and (3) all others. The order of presentation of the targets was randomized, and the subjects were instructed to shuffle the deck of 81 comparison figures after each sorting.

\section{Results}

The criterion used for finding triples of figures $(\mathrm{T}, \mathrm{C} 1, \mathrm{C} 2)$ with the proper class relationship (as in Figure 2) was based on the proportion of subjects who characterized a particular plain comparison figure (CX) as "easy to see the same sort of object in the figure as that portrayed by the target" $[\mathrm{P}(\mathrm{T}, \mathrm{CX})]$, for a particular drawn-in interpretation of the target. The classes shown in Figure 2, for example, include comparison figures for which

Table 1

Designations of All Target and Comparison Figures, Interpretation of Targets, and D Values for Eight Triples of Figures

\begin{tabular}{|c|c|c|c|}
\hline $\begin{array}{l}\text { Target } \\
\text { Figure* }\end{array}$ & Interpretation** & $\begin{array}{l}\text { Comparison } \\
\text { Figure }\end{array}$ & $D_{\dagger}^{\dagger}$ \\
\hline A8 & $\begin{array}{l}\text { Hippo Head Right } \\
\text { Human Face Left }\end{array}$ & $\begin{array}{l}\text { A1 } \\
17\end{array}$ & $.45(.14)$ \\
\hline B2 & $\begin{array}{l}\text { Animal Head Right } \\
\text { Gingerbread Man } 45^{\circ} \text { Left }\end{array}$ & $\begin{array}{l}\mathrm{A} 3 \\
\mathrm{C} 1\end{array}$ & $.77(.15)$ \\
\hline $\mathrm{C} 6$ & $\begin{array}{l}\text { Human Face Left } \\
\text { Butterfly } 45^{\circ} \text { Left }\end{array}$ & $\begin{array}{l}\text { B7 } \\
\text { C8 }\end{array}$ & $.42(.17)$ \\
\hline $\mathrm{E} 2$ & $\begin{array}{l}\text { Animal Head Left } \\
\text { Human Face Right }\end{array}$ & $\begin{array}{l}\mathrm{C} 2 \\
\mathrm{~F} 3\end{array}$ & $.71(.16)$ \\
\hline E6 & $\begin{array}{l}\text { Bird Head Right } \\
\text { Animal Head Left }\end{array}$ & $\begin{array}{l}\text { E8 } \\
\text { D5 }\end{array}$ & $.66(.16)$ \\
\hline F7 & $\begin{array}{l}\text { Animal Head Left } \\
\text { Open-Mouthed Beast Left }\end{array}$ & $\begin{array}{l}\text { F9 } \\
\text { G8 }\end{array}$ & $.37(.18)$ \\
\hline $\mathrm{G} 2$ & $\begin{array}{l}\text { Africa } \\
\text { Hippo Head Left }\end{array}$ & $\begin{array}{l}\text { G4 } \\
\text { G9 }\end{array}$ & $.54(.20)$ \\
\hline H8 & $\begin{array}{l}\text { Woman in Hat } \\
\text { Stone Axe }\end{array}$ & $\begin{array}{l}- \\
-\end{array}$ & - \\
\hline 15 & $\begin{array}{l}\text { Stone Axe } \\
\text { Gliding Bird }\end{array}$ & $\begin{array}{l}\mathrm{H} 4 \\
\mathrm{H} 9\end{array}$ & $.56(.21)$ \\
\hline
\end{tabular}

*Alphanumeric designations of figures correspond to the nine distinct rows and columns in Shepard and Cermak (1973).

**Interpretations listed first belonged to the first of target figures; those listed second belonged to the second set.

$\dagger D=\left\{\left[P(T, C 1)-P(T, C 2)_{\text {Inierp },+}+[P(T, C 2)-\right.\right.$

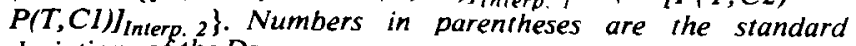
deviations of the Ds. 
$\mathrm{P}(\mathrm{T}, \mathrm{CX})>.30$. Given that each subject assigned about five figures to the "easy to see..." category, per target, then the $P(T, C X)$ expected for each figure $\mathrm{CX}$ on the basis of all figures having equal probability of being among the five chosen is on the order of .06. Thus, .30 is a highly nonrandom result. We wanted $P(T, C 1)>P(T, C 2)$ for one interpretation, but $P(T, C 1)<P(T, C 2)$ for the second interpretation. Eight triples of figures were found such that $\mathrm{D}=\left\{[\mathrm{P}(\mathrm{T}, \mathrm{C} 1)-\mathrm{P}(\mathrm{T}, \mathrm{C} 2)]_{\text {Interp. } 1}+[\mathrm{P}(\mathrm{T}, \mathrm{C} 2)-\right.$ $\mathrm{P}(\mathrm{T}, \mathrm{Cl})]_{\text {Interp. } 2\}}>.4$, where .4 is an entirely arbitrary criterion. Values of D are given in Table 1 , along with the standard deviation for each (calculated on the assumption that the assignment of a particular figure CX to the "easy to see..." class or not, by a single subject, constituted a Bernoulli trial; the number of trials per figure was the number of subjects). None of the members of any triple was a member of any other triple.

\section{EXPERIMENTS 2 AND 3: DELAYED COMPARISON DISCRIMINATION}

These experiments were designed to determine whether figures in the same interpretation class (defined in Experiment 1) would be discriminated more poorly than figures in different interpretation classes. Class membership was manipulated by changing the interpretation of the target figures. The two experiments used precisely the same stimulus set in the same presentation format, except that in Experiment 2 a delay of $13 \mathrm{sec}$ occurred between offset of the target figure and onset of the comparison figure, while in Experiment 3 that delay was $300 \mathrm{msec}$ in order to minimize memory effects. Also, in Experiment 2 the dependent measure was proportion correct, and in Experiment 3 response latency as well as accuracy data were collected. Otherwise, the experiments used the same procedure.

\section{Experiment 2}

\section{Method}

Stimuli and Apparatus. The stimuli were figures selected from the basic set of 81 according to rules specified in the Procedure section. Figures with added lines were the eight target figures satisfying the criterion of Experiment 1, or were modeled after the target figures of Experiment 1, in the case of Different and Same trials, respectively. All stimuli were $5 \times 5 \mathrm{~cm}$ black-onwhite drawings, and were presented via an Iconix three-field tachistoscope.

Subjects. Twenty-four paid adult female volunteers served as subjects. All were Stanford University undergraduates. None had served in Experiment 1.

Procedure. The subjects were told that each trial would consist of the presentation, for $3 \mathrm{sec}$, of an outline drawing with added interior lines as a target, a blank interval of $13 \mathrm{sec}$, and then the presentation of a plain outline drawing as a comparison. The comparison outline was to be considered the same as the target if it could be filled in to exactly match the target. An example is shown in Figure 1. A verbal response of "same" or "different" was required. It was stressed that Same trials and
Different trials would occur equally often and in a random sequence.

Each subject was presented a different sequence of 64 trials, 32 Same trials and 32 Different trials. The 32 Different trials were constructed from the eight validated triples of figures of Experiment 1. Four trials were produced from each triple: Each target had its two drawn interpretations, and each interpretation was presented with the two different comparison outline figures. The outline comparison figures (e.g., $\mathrm{C} 1, \mathrm{C} 2)$ were those found, in Experiment 1, to have the property that under one interpretation of the target (T), C1 is more easily interpreted (than $\mathrm{C} 2$ ) as representing the same sort of object as $T$; but under the second interpretation of $\mathrm{T}, \mathrm{C} 2$ is more easily interpreted (than $\mathrm{Cl}$ ) as the same kind of object as represented by $T$.

The stimuli used for Same trials were identical when used as the target and as the comparison figure except that the target contained the interior lines. The Same stimuli were chosen from outlines in the Shepard-Cermak set which had not been used in any of the 24 target and comparison figures of the Different trials. However, for each interpretive line drawing of a target appearing in Different trials, a similar interpretive drawing was used in at least one Same trial.' Table 1 lists the figures used in the Different trials (except H8), and the interpretations which were used in both Same and Different trials.

\section{Experiment 3}

Subjects. Twelve paid volunteers served as subjects. All but one were students at the University of Kansas, and nine were female.

Procedure. Each subject received a unique random ordering of 128 trials which included two replications of 32 Same trials and 32 Different trials (so that there were 1,536 subject trials, as in Experiment 2). The 64 unique trials included exactly the same target-comparison-interpretation combinations used in Experiment 2. Each trial consisted of the presentation of a drawn target for $3 \mathrm{sec}$, a 300-msec dark interval, then the presentation of a comparison figure which remained on until a response was made. The subjects were instructed to decide whether the comparison was the same as the target or different, and to respond with speed and accuracy in pushing a right or left button, respectively. "Same" was defined as applying to a comparison figure which could be made identical to the preceding target by the addition of extra lines to the basic outline. A series of 16 unscored warm-up trials preceded the experiment. None of the warm-up trial figures was used later.

\section{Results}

The basic result of Experiment 2 was that the judged ease of interpreting a comparison figure as the same sort of object as a target figure (the definition here of class membership) had a statistically reliable relationship to performance in the samedifferent delayed comparison discrimination task. Comparison figures which were different from the target but which could take the same interpretation were discriminated more poorly than comparison figures which could not easily be interpreted like the target. The result held even though parametric differences between the outlines of targets and comparison figures were equal whether they were within the same class or in different classes (betweenclass). Overall proportions correct for the three conditions were: Same, .78; Different, between, .70; Different, within, .59. Testing for differences between these proportions by computing a difference 
statistic for each subject, the (Same - Different, between) and (Different, between - Different, within) statistics were reliably positive: $t(23)=1.96$, $\mathrm{p}<.05$, and $\mathrm{t}(23)=2.60, \mathrm{p}<.01$, respectively.

The error data of Experiment 3 also revealed a difference between discrimination performance for the two types of Different comparison figures (within-class and between-class). The response time (RT) data, however, revealed no such differences. Overall proportions correct for the three conditions were: Same, .91; Different, between, .77; Different, within, .72. The difference in proportion correct between the Same condition and the Different, between, condition was shown reliable by a sign test $(p<.05)$. The effect of interest, the performance difference in the two Different conditions, was small but reliable statistically, $t(11)=1.91, \mathrm{p}<.05$ (onetailed).

The main feature of the RT data was their noisiness: There were $18 \%$ errors overall, a mean RT of $1,341 \mathrm{msec}$ for correct responses, and a standard deviation of $716 \mathrm{msec}$. No statistically reliable differences between conditions could be found with variability of that order. Even when the RT distributions for individual subjects were brought to a common origin by subtracting each subject's mean RT from all her scores, still there were no statistically reliable differences between the Same, Different, between, and Different, within, RT distributions (although the means of the distributions were ordered that way).

Despite the difference in target-comparison delay interval between the two experiments, the patterns of errors were similar. Proportions correct for the three conditions for both of the experiments are presented in Figure 3. Also, there were 32 different targetcomparison-interpretation combinations for Same trials and 32 combinations for Different trials. The proportion of errors for each combination was similar for the two delay intervals: The correlation of the error rates for the $\mathbf{3 2}$ Same figure combinations, between Experiments 2 and 3, was $r=.50$; for the Different figures the correlation between the two experiments was $r=.55$. These correlations are reliably nonzero: $t(30)=3.16, p<.01$, and $\mathrm{t}(30)=3.61, \mathrm{p}<.01$, respectively.

\section{GENERAL DISCUSSION}

The interpretation of a stimulus (as being an instance of $x$, as opposed to not being an instance of $x)$ is important as an independent variable, regardless of stimulus modality, when the interpretation variable is defined on single stimuli. The experiments here examined whether the interpretability of a visual stimulus would have an effect as an independent variable in a particular subclass of interpretation experiment in which the relationship of the inter-

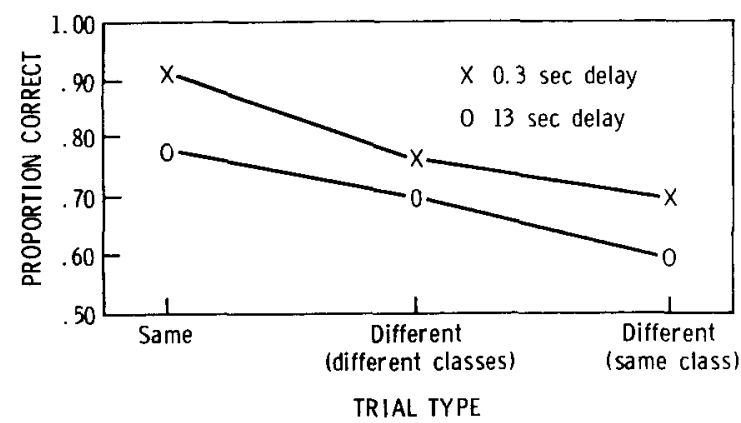

Figure 3. Proportion correct for Same pairs, Different pairs whose members were in the same class, and Different pairs whose members were in different classes.

pretability of two stimuli was the independent variable and confusability of the two stimuli was the dependent variable. It is concluded that the predicted relationship between the independent and dependent variables was observed: A pair of stimuli which people tended to interpret in the same way were confused more often than a pair of stimuli which people tended to interpret in different ways. Care was taken to ensure that members of the "sameinterpretation" pairs were as different physically as members of "different-interpretation" pairs. Many experiments using auditory stimuli support a conclusion at least as strong as the one reached here. It thus appears likely that the enhanced confusability of stimuli in the same interpretation-defined class is a general phenomenon, not limited to a particular sensory modality. Conclusions stronger than the one above may indeed be appropriate only to experiments employing certain types of stimuli and certain experimental procedures. The fact that the present effect is small compared to those observed using stop consonants could be due either to properties of the stimuli or to differences in experimental procedure. However, present conclusions are not based on the size of the effect, but on its existence.

The results of the present experiments do not entirely resolve the issue of whether the within-class confusion effect is a memory effect. The effect was apparently greater at an interstimulus delay of $13 \mathrm{sec}$ than at $300 \mathrm{msec}$, but the effect did appear at $300 \mathrm{msec}$ (see also Pisoni, 1973). Whatever one's view of the human information-processing system, it seems safe to say that the elements of the system which are relatively independent of time delays (at least on the order of 10-20 sec) are the ones primarily responsible for the within-class confusion effect, although time-dependent elements may increase the effect.

It is not surprising that interpretation should have an effect in two-stimulus confusability studies. It would be more surprising if no effect were ob- 
served. The interesting question may be, instead, what it is about the structure of individual stimuli, and the differences among stimuli within some physically defined group, that leads to rather abrupt and disjoint classes of stimuli being defined by human observers.

\section{REFERENCES}

Bower. G. H. . \& HolyoAk, K. Encoding and recognition memory for naturalistic sounds. Journal of Experimental Psychology, 1973. 101. 360-366.

Bower, G. H.. Karlin, M. B., \& Dueck, A. Comprehension and memory for pictures. Memory \& Cognition, 1975, 3. 216-220.

Bugelski, B. R., \& AlaMPay, D. A. The role of frequency in developing perceptual sets. Canadian Journal of Psychology, $1961,15,205-211$.

Cross, D. V., Lane, H. L., \& She ppard, W. C. Identification and discrimination functions for a visual continuum and their relation to the motor theory of speech perception. Journal of Experimental Psychology, 1965, 70, 63-74.

Cutting. J. E., \& Rosner. B. S. Categories and boundaries in speech and music. Perception \& Psychophysics, 1974, 16. 564-570.

Curting, J. E.. \& Rosner, B. S. Discrimination functions predicted from categories in speech and music. Perception \& Psychophysics, 1976, 20, 87-88.

GARNER, W. R. Information integration and form of encoding. In A. W. Melton \& E. Martin (Eds.). Coding processes in human memor. Washington, D.C: Winston, 1972.

HoснвERG. J. In the mind's eye. In R. N. Haber (Ed.). Contemporan theory and research in visual perception. New York: Holt, Rinetiart. \& Winston, 1968.

LANE. $H$. The motor theory of speech perception: A critical review. Psichological Review. 1965. 72. 275-309.

LENNEBERG, E. Color naming, color recognition, color discrimination: A reappraisal. Perceptual and Motor Skills, 1961, 12, 375-382.

liberman, A. M., Cooper, F. S., Shankweiler, D. P., \& Studdert-Kennedy, M. Perception of the speech code. Psychological Review, 1967, 74, 431-461.

Lib erman, A. M., Harris, K. S., Hoffman, H. S., \& Griffith, $B$. C. The discrimination of speech sounds within and across phoneme boundaries. Journal of Experimental Psychology. 1957. 54. 358-368.
Liberman, A. M., Mattingly, I. G. \& \& Turvey, M. T. Language codes and memory codes. In A. W. Melton \& E. Martin (Eds.), Coding processes in human memory. Washington. D.C: Winston. 1972.

Mattingly, I. G., Liberman. A. M., Syrdal, A. K.. \& Halwes, T. Discrimination in speech and nonspeech modes. Cognitive Psychology, 1971, 2, 131-157.

Pison, D. B. Auditory and phonetic memory codes in the discrimination of consonants and vowels. Perception \& Psychophysics, 1973, 13, 253-260.

Pisoni, D. B. Auditory short-term memory and vowel perception. Memony \& Cognition, 1975, 3, 7-18.

Pisoni. D. B., \& TASH, J. Reaction times to comparisons within and across phonetic categories. Perception \& Psychophysics. 1974, 15. $285-290$.

RILEY. D. A. Memory for form. In L. Postman (Ed.), Psychology in the making. New York: Knopf, 1962.

Shepard, R. N., \& Cermak, G. W. Perceptual-cognitive explorations of a toroidal set of free-form stimuli. Cognitive Psychology. 1973. 4, 351-377.

Studdert-Kennedy, M., Liberman, A. M., Harris, K. S., \& COOPER, F. S. Motor theory of speech perception: A reply to Lane's critical review. Psychological Review. 1970. 77. 234-249.

Uhr. L. Pattern recognition. In L. Uhr (Ed.), Pattern recognition. New York: Wiley. 1966.

\section{NOTE}

1. Each of the 16 distinct interpretive drawings in the Different target set appeared twice. For 10 of these 16 , it was possible to find two other outlines which could be given a similar interpretive drawing for use in Same trials. For the remaining six, only one outline could be found. Three interpretive drawings not used in the Different trials were applied to six other outlines to complete the set of 32 Same trials. Thus, frequency of particular interpretations for Same and Different trials was similar but not identical. Twenty-one different outlines were used to produce the Same-trial figures. Eleven were used twice (with different interpretive drawings each time) and 10 were used once. In the data analysis, important comparisons are made within the Different trials, not between Different and Same trials.

(Received for publication June 14, 1976; revision accepted November $25,1976$. 